\title{
Hyperintense Vessels on Magnetic Resonance Imaging Correlate with Arteries in the Delayed Phase of Multiphase Computed Tomography Angiography
}

\author{
Hye Jeong Lee, MD (D); Yo Han Jung, MD, PhD (iD; Kyung-Yul Lee, MD, PhD (D) \\ Department of Neurology, Gangnam Severance Hospital, Yonsei University College of Medicine, Seoul, Korea
}

Collateral circulation plays an important role in protecting brain tissue from ischemic damage in acute cerebral artery occlusion, and various imaging modalities are used to evaluate collateral circulation. Angiographic imaging techniques, such as digital subtraction angiography and multiphase computed tomography (CT) angiography, directly show cerebral collateral circulation. Meanwhile, hyperintense vessels on fluid-attenuated inversion recovery and T1-weighted black-blood fast spin echo magnetic resonance imaging (MRI) are known to indirectly reflect collateral circulation in cases of cerebral artery occlusion. Herein, we compare hyperintense vessels on MRI with collateral circulation on multiphase CT angiography in a patient with acute ischemic stroke with occlusion of the middle cerebral artery.

J Neurosonol Neuroimag 2021;13(1):14-17

Key Words: Blood vessels; Magnetic resonance imaging; Stroke
Received: February 9, 2021

Revised: April 1, 2021

Accepted: April 13, 2021

Correspondence: Kyung-Yul Lee, MD, PhD

Department of Neurology, Gangnam Severance Hospital, Yonsei University College of Medicine, 211 Eonju-ro, Gangnam-gu, Seoul o6273, Korea

Tel: +82-2-2019-3325

Fax: +82-2-3462-5904

E-mail:kylee@yuhs.ac
In acute cerebral artery occlusion, the collateral circulation plays an important role in preserving ischemic penumbra volume, prolonging the therapeutic time window, and slowing the infarction progression rate. ${ }^{1}$

Several imaging modalities have been used to evaluate the collateral circulation. The gold standard method is digital subtraction angiography, but recently there have been several reports on the clinical usefulness of multiphase computed tomography (CT) angiography to evaluate collateral circulation. ${ }^{2}$ The delayed phase of multiphase CT angiography demonstrates that collateral circulation slowly enhances vessels by making them retrograde opacifications via pial collaterals. As a result, arteries in the delayed phase of CT angiography are well correlated with leptomeningeal collateral grade on conventional angiography in acute ischemic stroke. ${ }^{3}$

Meanwhile, hyperintense vessels on fluid-attenuated inversion recovery (FLAIR) are known to reflect collateral blood flow in cerebral artery occlusion. ${ }^{4}$ Furthermore, hyperintense vessels are well correlated with hyperintense vessels on three-dimensional T1-weighted blackblood fast spin echo magnetic resonance imaging (MRI). ${ }^{5}$
We present the case of a patient with acute ischemic stroke with occlusion of the inferior division of the middle cerebral artery. This case demonstrates a correlation between collateral vessels on multiphase CT angiography and hyperintense vessels on MRI.

\section{CASE}

An 82-year-old woman was admitted because of a language disturbance and right-sided weakness. She suddenly noticed that she could not speak while having dinner.

She had a history of pancreatic cancer in 2006 and achieved complete remission after a Whipple pancreaticoduodenectomy with adjuvant chemoradiation therapy. Diabetes mellitus developed after a Whipple operation. In 2019, she was diagnosed with peritoneal carcinomatosis, causing ascites and dyspnea on exertion. Carcinomatosis was considered to be metastasis of other primary cancers rather than recurrence of the previous pancreatic cancer. She received the 8th gener- 
ation chemotherapy with pembrolizumab and underwent paracentesis weekly after chemotherapy.

Her initial neurologic examination revealed grade IV right-sided arm weakness, right facial palsy, severe dysarthria, and aphasia. The National Institutes of Health Stroke Scale (NIHSS) scores were 8. Brain multiphase CT angiography performed 2.5 hours after symptom onset showed occlusion of the inferior division of the left middle cerebral artery in the first arterial phase. The second and third phases of multiphase CT angiography revealed a delayed appearance of the left middle cerebral artery (Fig. 1A-C). These images are indicative of one phase delay in filling of peripheral vessels, with similar extent and prominence when compared with the contralateral hemisphere. ${ }^{2}$ Brain MRI, which was performed 3 hours after symptom onset, revealed hy- perintense vessels on T1-weighted black-blood fast spin echo MRI and FLAIR, and they were well correlated with arteries seen on the delayed phase of multiphase CT angiography (Fig. 1D-F). Diffusion-weighted and perfusion-weighted images showed diffusion-restrictive lesions in the left parieto-temporal lobe (Fig. 2A) combined with perfusion delay (Fig. 2). Intra-arterial thrombectomy was not performed because of distal branch occlusion and terminal status of the cancer.

Laboratory test results were normal except for the presence of anemia ( $\mathrm{Hb} 9.1 \mathrm{~g} / \mathrm{dL})$ and high d-dimer (4.33 $\mu \mathrm{g} / \mathrm{mL}$, normal o-0.65 $\mu \mathrm{g} / \mathrm{mL}$ ) and HbAic (8.4\%) levels. Electrocardiogram and transthoracic echocardiography findings were normal.

Follow-up MRI 3 days after stroke onset revealed expanded diffusion-restricted lesions (Fig. 2C) with de-
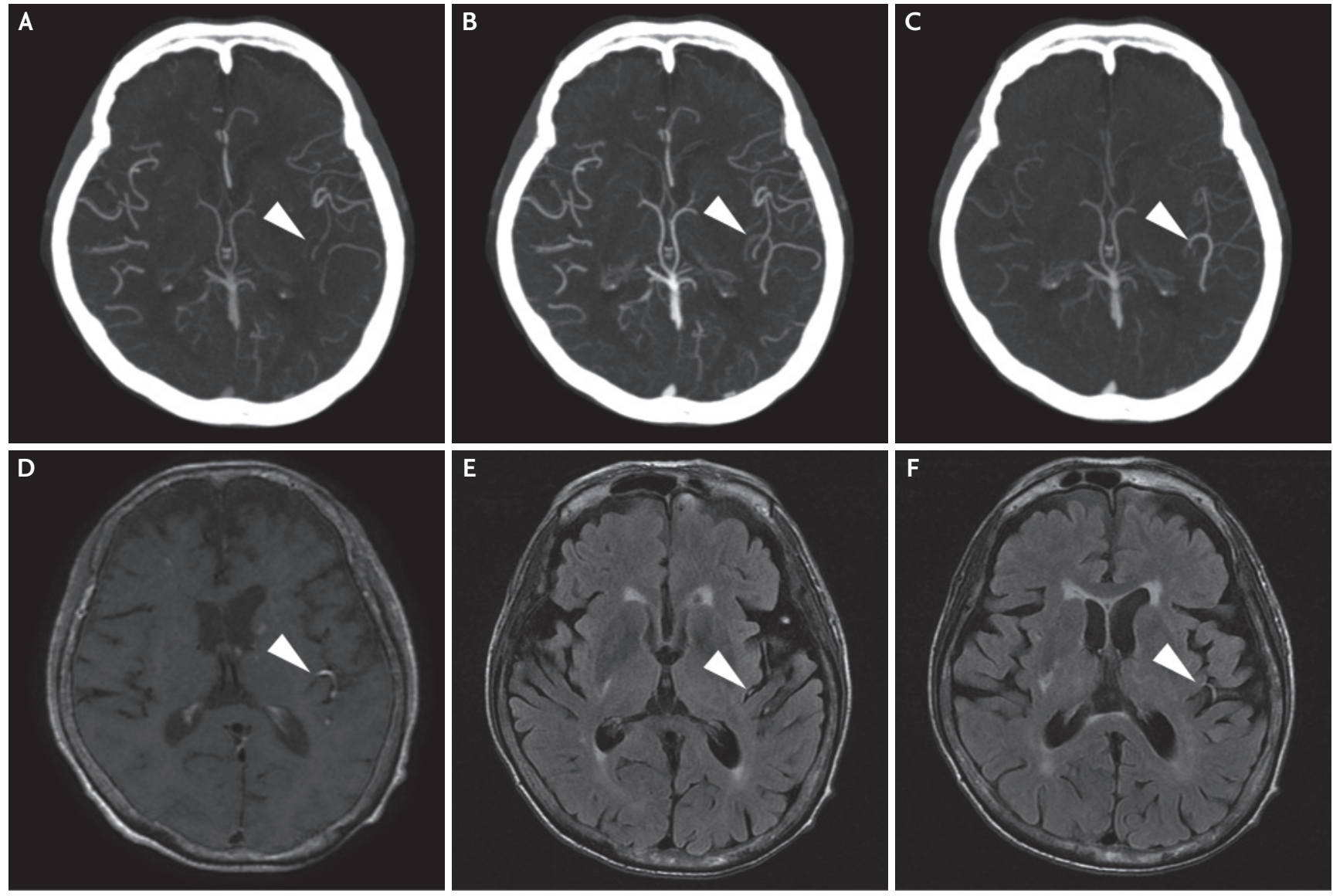

FIG. 1. Brain computed tomography (CT) angiography and magnetic resonance imaging of an 82-year-old woman with aphasia and right-sided weakness. (A-C) Axial maximum intensity projection images of brain CT angiography demonstrate that the distal branch of the left middle cerebral artery (arrowhead) is rarely visualized on early phase (A) CT angiography and (B, C) is well visualized on delayed phase CT angiography. These results indicate a pial arterial filling score of 4 . (D) This distal branch of the middle cerebral artery is matched with a high-signal intensity vessel on three-dimensional T1-weighted black-blood fast spin echo and (E, F) fluid-attenuated inversion recovery images. 
creased number and intensity of FLAIR hyperintense vessels (Fig. 2D), and MRA revealed partial recanalization with a paucity of vessels in the left middle cerebral artery (Fig. 2E). The patient's symptoms dramatically improved (NIHSS score, o). The Institutional Review Board waived the need for informed consent (IRB No. 3-2020-0476).

\section{DISCUSSION}

Adequate cerebral collateral circulation plays an important role in preventing ischemic damage in cerebral arterial occlusion by maintaining blood flow to the penumbral tissue in the absence of revascularization. In addition, good collateral circulation prolongs the therapeutic time window, slows the progression of infarct growth, and reduces hemorrhagic transformation during intravenous and intra-arterial thrombolytic treatment. $^{1}$

Conventional cerebral angiography is the gold standard method to assess leptomeningeal collateral status; however, it is time consuming and requires specialized clinicians to perform. Therefore, various neuroimaging modalities, such as multiphase CT angiography and time-resolved MR angiography, are used to evaluate collateral circulation in acute stroke management. ${ }^{6}$

Multiphase CT angiography is useful for evaluating the overall cerebral perfusion and collateral status. It provides sequential images allowing temporal- and spatial-resolved assessment in addition to the prominence and extent of vessels detectable with the single phase CT angiography. ${ }^{2}$ Furthermore, Alberta Stroke Program Early CT Score assessment using multiphase $\mathrm{CT}$ angiography has been known to be more sensitive than non-contrast CT in the early detection of ischemic areas and prediction of final infarct size. ${ }^{7}$ Collateral status is visually assessed by comparing asymmetric retrograde filling arteries beyond the occluded or highgrade stenotic arteries with similar arteries in the contralateral hemisphere. Therefore, the delayed phase of multiphase CT angiography improves the speed and accuracy of interpretation in distal anterior circulation occlusions, even in non-stroke specialists. ${ }^{8}$
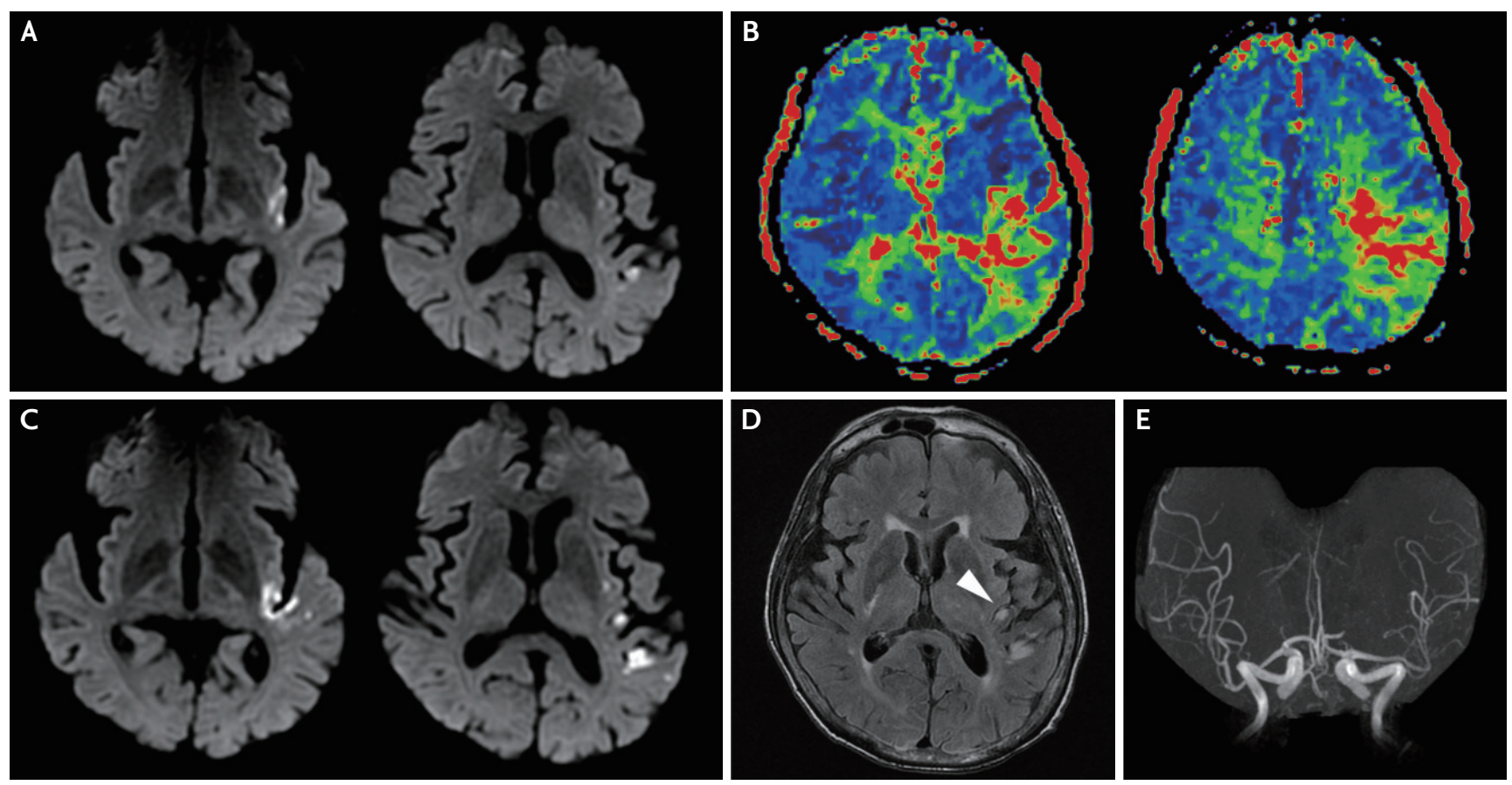

FIG. 2. (A, B) Initial brain diffusion and perfusion weighted magnetic resonance imaging and (C-E) follow up fluid-attenuated inversion recovery (FLAIR) image and magnetic resonance angiography (MRA). (A) Brain diffusion-weighted images show acute ischemic stroke in the left temporal lobe. (B) Mean transit time perfusion weighted images show perfusion delay in the left parieto-temporal lobe. (C) Follow-up brain diffusion-weighted images showing lesion expansion. (D) Hyperintense vessels (arrowhead) are decreased in the follow-up FLAIR, and (E) there was a paucity of collaterals in the left middle cerebral artery on follow-up MRA. 
Hyperintense vessels on FLAIR and three-dimensional T1-weighted black-blood fast spin echo imaging are defined as linear high signal intensities along with vessel courses most conspicuous in the Sylvian fissure and cortical sulci. They have been suggested as indirect findings of slow anterograde flow or retrograde leptomeningeal collaterals. Although their significance remains unclear, hyperintense vessels are prominently observed in patients with acute cerebral infarction accompanied by intracranial arterial occlusion or significant stenosis of the anterior cerebral circulation. Some studies suggest that hyperintense vessels are associated with adequate collateral flow, so the presence of hyperintense vessels on MRI may imply good functional outcome in acute cerebral infarction with collateral ciculations. ${ }^{9}$ Sakuta et al. ${ }^{10}$ reported that a decrease number and intensity of hyperintense vessels on FLAIR in hyperintense vessels on FLAIR in patients with acute ischemic stroke following administration of tissue plasminogen activator therapy showed better functional outcomes than those without a decrease in hyperintense vessels.

In this case, collateral vessels were seen as hyperintense vessels on FLAIR and three-dimensional T1-weighted black-blood fast spin echo MRI, and these vessels were seen on the delayed phase of multiphase CT angiography. In addition, the perfusion delay area on the mean transit time map of the perfusion weighted image correlated well with hyperintense vessels. Therefore, we may measure collateral and cerebral perfusion status using multiphase CT angiography rather than MRI.

\section{Conflicts of Interest}

No potential conflicts of interest relevant to this article was reported.

\section{REFERENCES}

1. Bang OY, Goyal M, Liebeskind DS. Collateral circulation in ischemic stroke: assessment tools and therapeutic strategies. Stroke. 2015;46:3302-3309.

2. Menon BK, d'Esterre CD, Qazi EM, Almekhlafi M, Hahn L, Demchuk AM, et al. Multiphase CT angiography: a new tool for the imaging triage of patients with acute ischemic stroke. Radiology. 2015;275:510-520.

3. Maier IL, Scalzo F, Leyhe JR, Schregel K, Behme D, Tsogkas I, et al. Validation of collateral scoring on flat-detector multiphase CT angiography in patients with acute ischemic stroke. PLoS One. 2018;13:e0202592.

4. Lee KY, Latour LL, Luby M, Hsia AW, Merino JG, Warach S. Distal hyperintense vessels on FLAIR: an MRI marker for collateral circulation in acute stroke? Neurology. 2009;72:1134-1139.

5. Lee KY, Suh SH, Ahn SJ. Significance of hyperintense arteries on Gd-enhanced 3D T1W black-blood imaging in acute stroke. Eur Radiol. 2019;29:1329-1337.

6. Balaji R. Time-resolved MR angiography in wake-up stroke: an innovative application of a proven technique. Acad Radiol. 2015;22:411-412.

7. Sallustio F, Motta C, Pizzuto S, Diomedi M, Rizzato B, Panella M, et al. CT angiography ASPECTS predicts outcome much better than noncontrast CT in patients with stroke treated endovascularly. AJNR Am J Neuroradiol. 2017;38:1569-1573.

8. Yu AY, Zerna C, Assis Z, Holodinsky JK, Randhawa PA, Najm $\mathrm{M}$, et al. Multiphase CT angiography increases detection of anterior circulation intracranial occlusion. Neurology. 2016;87:609-616.

9. Schellinger PD, Chalela JA, Kang DW, Latour LL, Warach S. Diagnostic and prognostic value of early MR imaging vessel signs in hyperacute stroke patients imaged $<3$ hours and treated with recombinant tissue plasminogen activator. AJNR Am J Neuroradiol. 2005;26:618-624.

10. Sakuta K, Saji N, Aoki J, Sakamoto Y, Shibazaki K, Iguchi Y, et al. Decrease of hyperintense vessels on fluid-attenuated inversion recovery predicts good outcome in t-PA patients. Cerebrovasc Dis. 2016;41:211-218. 